\title{
Propiedades Psicométricas de la Escala de Negatividad Social de Newsom en Universitarios Chilenos
}

\section{Psychometric Properties of Newson's Social Negativity Exchange Scale in a Chilean College Age Students}

\author{
Manuel S. Ortiz \\ Departamento de Psicología, Universidad de La Frontera, Chile. \\ Daniela Gómez-Pérez \\ Programa de Doctorado en Psicología, Universidad de La Frontera, Chile. \\ Margarita Cancino \\ Programa de Doctorado en Psicología, Universidad de La Frontera, Chile. \\ Ana Barrera-Herrera \\ Programa de Doctorado en Psicología, Universidad de La Frontera, Chile. \\ Rec (15 de marzo de 2016) Acept (2 de marzo de 2017)
}

\begin{abstract}
Resumen
El objetivo de este estudio fue estimar las propiedades psicométricas de la Escala de Negatividad Social de Newsom, la cual mide el efecto negativo de las interacciones sociales en el bienestar individual. Para ello, se seleccionó a 526 universitarios, realizando análisis factorial exploratorio con 50\% de la muestra y análisis factorial confirmatorio con el restante $50 \%$. A diferencia de la estructura de cuatro factores sugerida por los autores, los resultados de este estudio indican que la escala tiene una estructura factorial de dos factores, agrupando el primer factor 4 ítems y el segundo 8. Las cargas factoriales fueron superiores a 0,3 y se obtuvieron buenos indicadores de bondad de ajuste. La consistencia interna de la escala, medida con Alfa de Cronbach fue 0,86. La evidencia sugiere que la Escala de Negatividad Social de Newsom es un instrumento válido y confiable que puede ser utilizado en población Chilena.

Palabras clave: Negatividad Social, Análisis Factorial Exploratorio, Análisis Factorial Confirmatorio, Confiabilidad.
\end{abstract}

\begin{abstract}
The purpose of this study was to determine the psychometric properties of the Social Negativity Exchange Scale developed by Newsom et al, which is defined as the negative effect that social interactions have on individual wellbeing. Thus, a sample of five hundred and twenty two $(n=526)$ college-age students was nonrandomly selected. An exploratory factor analysis was conducted with $50 \%$ of them, and then a confirmatory factor analysis with the others. A two-factor solution was identified, with the first factor grouping four items and the second factor eight items. Fit indexes were good, with factorial loadings greater than .3. The total scale reliability was .86. The evidence obtained in this study suggests that the Newsom's Social Negativity Exchange Scale is a valid and reliable scale to be used with Chilean population.

Key words: Social Negativity, Exploratory Factorial Analysis, Confirmatory Factorial Analysis, Reliability.
\end{abstract}




\section{Introducción}

En el estudio de las relaciones sociales, la perspectiva dominante es aquella que enfatiza que estas producen mejores resultados en la salud física y mental de las personas. Desde esta mirada, disponer de una mayor cantidad de vínculos sociales se asocia a mejores resultados en salud, pues se entiende que las interacciones sociales favorecen la búsqueda y recepción de apoyo social (Årestedt, Saveman, Johansson y Blomqvist, 2013), se asocian a estilos de afrontamiento saludables y moderan los efectos del estrés psicológico (Ortiz, Willey y Chiang, 2014). Sin embargo, un aspecto menos estudiado son las consecuencias negativas o no deseadas que estas pueden generar. Así, diversos estudios señalan que los intercambios sociales negativos pueden inducir altos niveles de estrés psicológico, reducir la percepción de satisfacción social de los individuos y generar negatividad social (Barra, 2014; Ibarra-Rovillard y Kuiper, 2011; Newsom, Mahan, Rook y Krause, 2008; Rook, 2014).

Al respecto, la negatividad social (NS), es un constructo multidimensional definido como el efecto negativo que las relaciones sociales tienen en el bienestar individual (Ibarra-Rovillard y Kuiper, 2011) y que resulta de conductas que son percibidas por el receptor como aversivas y/o no deseadas (Newsom, Nishishiba, Morgann, y Rook, 2003). Estos intercambios sociales pueden incluir elementos como conflicto, rechazo, crítica o intrusión (Newsom et al., 2008; Rook, 2014). De acuerdo a Brooks y Dunkel-Schetter (2011), la NS incluye tres aspectos: (1) conflicto, que es definido como los comportamientos que provocan diferencias con otros y que se asocian con emociones como el enojo o ira; (2) insensibilidad, que alude a comportamientos de desprecio que otras personas tienen de las necesidades y deseos propios; (3) interferencia, que se refiere a comportamientos de otros que dificultan la consecusión de objetivos personales. Similarmente, Vinokur y Van Ryn (1993) identifican tres dimensiones de NS: (1) experimentación de afecto negativo (enojo o disgusto); (2) evaluación negativa que otros hacen de la persona en términos de sus atributos, acciones o esfuerzos (crítica); (3) intromisión en el logro de los objetivos instrumentales de la persona.

Además, otros estudios destacan la relevancia de la NS en contextos de salud; por ejemplo, la evidencia señala que se asocia con pobres autorreportes de salud (Newsom et al., 2008; Walen y Lachman, 2000), incidencia de enfermedad cardiovascular (De Vogli, Chandola, y Marmot, 2007), mayor reactividad cardiaca (Smith et al., 2009) y una respuesta inflamatoria exhacerbada (Carpenter, Gawuga, Tyrka, Lee,
Anderson y Price, 2010; Miller, Chen, y Zhou, 2007; Taylor, Lehman, Kiefe, y Seeman, 2006).

Dada la implicancia anteriormente reportada, es relevante disponer de un instrumento válido y confiable que permita medir este constructo en población Chilena. Para tal efecto, el presente estudio tiene por objetivo estimar las propiedades psicométricas de la escala de NS de Newsom, Rook, Nishishiba, Sorkin y Mahan (2005), que corresponde a uno de los instrumentos más utilizados para medir el constructo y que, a diferencia de las definiciones inicialmente propuestas, evalúa la NS a través de cuatro dimensiones: (1) instrusión, que corresponde a una interacción social caracterizada por la supervisión y/o intrusión; (2) falla en proporcionar ayuda; (3) comportamiento antipático/insensible, que hace referencia a una interacción social en donde existe escasa sensibilidad por las necesidades del otro y (4) rechazo y/o negligencia en una interacción social.

\section{Método}

\section{Participantes}

Por medio de un muestreo no probabilístico, por conveniencia, se seleccionó una muestra de 526 estudiantes de carreras de pregrado de la Universidad de La Frontera. La edad promedio de los participantes fue de 21,03 años (D.T. $=2,89$ ); el $66 \%$ de la muestra son mujeres y un $48 \%$ se identifica a sí mismo como perteneciente a la etnia Mapuche. Además, los estudiantes que conformaron la muestra tenían en promedio 2.32 años de antigüedad en la universidad (D.T. $=1,12$ ), y en su mayoría pertenecían a la carrera de Psicología (30\%).

\section{Instrumentos}

Escala de Negatividad Social (Newsom et al., 2005). Compuesta por 12 ítems que evalúan cuatro dimensiones de NS (Intrusión, Falla en proporcionar ayuda, Insensibilidad y Rechazo), con tres ítems para cada dimensión; su formato de respuesta es de tipo Likert, considerando cinco opciones que miden el grado de acuerdo o desacuerdo del sujeto con cada afirmación (ver Anexo 1), en donde a mayor puntaje mayor nivel de NS. Ante la ausencia de una versión en español de esta escala, se utilizó el procedimiento de back translation (Brislin, 1970), el cual es frecuentemente utilizado en estudios de carácter transcultural y cuyo objetivo 
es obtener una versión equivalente a la versión original al momento de traducir un instrumento.

Covariables. Diversas variables tales como el género, edad, estado civil, religión, nivel socioeconómico, pertenencia a un grupo étnico, entre otras, fueron evaluadas con un cuestionario sociodemográfico ad-hoc.

\section{Procedimiento}

Los participantes de este estudio fueron contactados en sus salas de clases, antes de comenzar el periodo lectivo, por estudiantes del Programa de Doctorado en Psicología de la Universidad de La Frontera, quienes explicaron los objetivos del estudio y los invitaron a participar. Aquellos que voluntariamente accedieron a participar de la investigación, firmaron un consentimiento informado que detalla objetivos del estudio, carácter voluntario, confidencial y anónimo de su participación, entre otros aspectos.

Este estudio fue aprobado por el Comité de Ética Científica de la Universidad de La Frontera.

\section{Plan de análisis}

Para cumplir con el objetivo de este estudio, la muestra fue aleatoriamente divida en dos. Con el $50 \%$ de la muestra se realizó Análisis Factorial Exploratorio (AFE) con extracción de componentes principales, rotación varimax, estimación de la medida de adecuación muestral de Kaiser Meyer Olkin (KMO) y la prueba de esfericidad de Bartlett. En segundo lugar, la solución factorial obtenida a través del AFE fue confirmada con un Análisis Factorial Confirmatorio (AFC; Schreibe, Nora, Stage, Barlow, y King, 2006) efectuado con el 50\% restante de la muestra, siguiendo la teoría de Bentler y Weeks (1980). Debido a que el supuesto de normalidad multivariada no fue asumido, la estimación de los modelos fue realizada con la corrección de Satorra-Bentler, incluyendo Satorra-Bentler Chi-Cuadrado (SB $X^{2}$ ), el Índice de Ajuste Comparativo (CFI > 0,95), Índice de Tucker Lewis (TLI > 0,90), el Error Cuadrático Medio de Aproximación $($ RMSEA $\leq 0,06)$ y la Media Estandarizada de la Raíz Cuadrática Residual (SRMR $<0,05$ ). Adicionalmente, el Test de Lagrange fue ejecutado de acuerdo a consideraciones teóricas y empíricas.

La consistencia interna de la escala fue estimada usando el estadístico Alfa de Cronbach.

Los análisis fueron ejecutados con el programa estadístico Stata 14.1 (StataCorp LP), considerando un alfa nominal $\leq 0,05$.

\section{Resultados}

\section{Análisis Factorial Exploratorio}

Preliminar al AFE, se estimó el índice de adecuación muestral (KMO) y el supuesto de esfericidad de Bartlett. El KMO obtenido es considerado un valor bueno $(\mathrm{KMO}=$ ,88), y la prueba de esfericidad resultó ser estadísticamente significativa $\left(X_{(66)}^{2}=1146,01 ; p \leq .0001\right)$, sugiriendo que el análisis factorial es una técnica adecuada para estos datos.

A continuación se realizó un AFE, el cual permitió identificar sólo dos factores. El primero de ellos tuvo un Eigenvalue de 4,7, agrupó ocho ítems y explicó el 36\% de la varianza. El segundo factor con un Eigenvalue de 1,65 , agrupó cuatro reactivos y dio cuenta del $17 \%$ de la varianza. En conjunto, ambos factores explicaron el 53\% de la varianza total de la escala. Todas las cargas factoriales fueron mayores a 0,3 (ver Tabla 1 ).

\section{Análisis Factorial Confirmatorio}

Para confirmar la solución obtenida previamente, se procedió a ejecutar un AFC. Debido al no cumplimiento del supuesto de normalidad multivariada de Mardia ( $p$ $\leq 0,001$ ), se utilizó la corrección de Satorra-Bentler. Tal como se observa en la Figura 1, la solución inicial de dos

Tabla 1. Cargas Factoriales de items en su respectivo factor

\begin{tabular}{ccc}
\hline Ítems & Factor 1 & Factor 2 \\
\hline 1 & & 0.68 \\
2 & & 0.74 \\
3 & 0.66 \\
4 & 0.76 & \\
5 & 0.39 & \\
6 & 0.77 & \\
7 & 0.70 & \\
8 & 0.77 & \\
9 & 0.78 & \\
10 & 0.83 & \\
11 & 0.57 & \\
12 & & \\
\hline
\end{tabular}

Fuente: elaboración propia. 
Figura 1. Solución factorial de la Escala de Negatividad Social de Newsom et al.

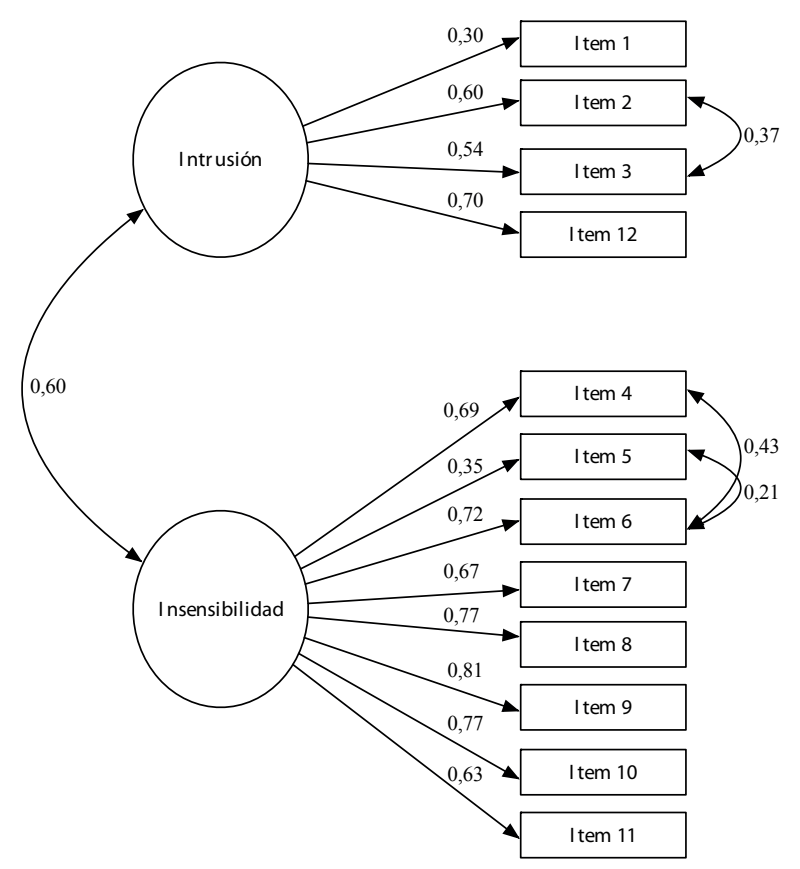

$\mathrm{SB}^{2}{ }_{(50)}=99,57 ; \mathrm{p} \leq 0,001 ; \mathrm{RMSEA}=0,06 ; \mathrm{CFI}=0,95 ; \mathrm{TLI}=0,93 ; \mathrm{SRMR}=0,056$

factores fue confirmada, con los mismos ítems cargando en los mismos factores y con pesos factoriales mayores a 0,3 . No obstante lo anterior, y posterior a la ejecución del Test de Lagrange, se efectuó el análisis incorporando al modelo la covarianza entre los dos factores y otras covarianzas entre algunos ítems. Con todo, los indicadores de bondad de ajuste del modelo fueron muy buenos $\left(\operatorname{SBX}^{2}(50)=\right.$ 63,$37 ; \mathrm{p} \leq 0,001 ; \mathrm{RMSEA}=0,032 ; \mathrm{CFI}=0,98 ; \mathrm{TLI}=0,98$; $\mathrm{SRMR}=0,053)$. La solución obtenida explica un $95 \%$ de la varianza $\left(\mathrm{R}^{2}=0,95\right)$.

En cuanto a la consistencia interna de la Escala, el Alfa de Crobbach fue 0,86 , lo cual refleja una buena confiabilidad del instrumento.

\section{Discusión}

La Negatividad Social es un constructo multidimensional estable, que hace referencia al efecto negativo de la interacción social en el bienestar de las personas (Ibarra-Rovillard y Kuiper, 2011). Esta variable tiene relevancia en el ámbito de la salud, debido a la asociación que tiene con factores psicológicos tales como el apoyo social percibido, depresión y estrés psicológico (Beach, Katz, Kim y Brody, 2003; Bertera, 2005; Ibarra-Rovillard y Kuiper, 2011). Sin embargo, y a diferencia de otros contructos psicológicos tales como el optimismo disposicional (Ortiz, Gómez-Pérez, Cancino y Barrera-Herrera, 2016), depresión y ansiedad (Antúnez y Vinet, 2012) y estrés (Ortiz, Baeza-Rivera y Myers, 2013), que cuentan con escalas validadas en población Chilena, las propiedades psicométricas de la escala de NS de Newsom et al. (2005) aún no han sido estudiadas en Chile.

Los resultados de este estudio, permiten afirmar que la mencionada escala cuenta con buenas propiedades psicométricas para medir el constructo de NS de forma válida y confiable en población Chilena. Sin embargo, a diferencia de la estructura factorial propuesta por Newsom et al. (2005), en este estudio se ha identificado una estructura de dos factores. Del análisis semántico de los ítems y del factor en el cual cargan, se propone denominar al primero de ellos como "Intrusión", por cuanto sus cuatro reactivos hacen referencia a interacciones sociales críticas e inoportunas ("le dio consejos que no necesitaba"). El nombre propuesto para el segundo factor es "Insensibilidad", el cual se compone de ocho ítems que aluden a intercambios negligentes e indiferentes de parte de un tercero ("fue poco considerado/a con usted").

Aún cuando los factores identificados en este estudio están moderadamente correlacionados, este resultado es similar a los obtenidos en investigaciones previas, los cuales indican que pese la existencia de esta asociación, las dimensiones que conforman el concepto son diferentes y por tanto hacen de la NS un constructo multidimensional (Brooks y Dunkel-Schetter, 2011; Finch, Okun, Pool y Ruehlman, 1999; Ruehlman y Karoly, 1991).

Este estudio posee algunas limitaciones. La primera de ellas guarda relación con que la muestra estudiada fue conformada por universitarios, por lo que se debe ser cauteloso al extrapolar estos resultados a la población general; es deseable que futuros estudios amplíen esta muestra a otros grupos etáreos. Una segunda limitación tiene que ver con que la escala no identifica las fuentes de NS, por lo que no es posible reconocer quienes la provocan, reduciendo con esto la posibilidad de distinguir qué personas podrían ser agentes generadores de estrés.

Finalmente, para futuros estudios, será importante realizar estudios de validez concurrente con otros constructos tales como sintomatología depresiva, apoyo social o estrés psicológico. Es altamente interesante estimar la asociación que la NS tiene con resultados de salud física y mental.

Dicho esto, la escala de NS de Newson et al. (2005), validada en este estudio posee una adecuada confiabilidad e identifica dos factores relacionados de NS, los cuales explican el $95 \%$ de la varianza del constructo. Contar con este instrumento validado permite su uso en ámbitos de 
salud y de investigación psicológica. La medición válida y confiable de este constructo, además favorece la planificación de tratamientos médicos que consideren la calidad de las relaciones e interacciones sociales de las personas, $\mathrm{o}$ planificar estrategias que permitan desarrollar redes de apoyo con interacciones positivas, a fin de fortalecer la prevención o recuperación de enfermedades agudas y/o crónicas.

\section{Referencias}

Antúnez, Z., y Vinet, E. V. (2012). Escalas de depresión, ansiedad y estrés (DASS-21): validación de la versión abreviada en estudiantes universitarios chilenos. Terapia psicológica, 30, 49-55.

Årestedt, K., Saveman, B. I., Johansson, P., y Blomqvist, K. (2013). Social support and its association with health-related quality of life among older patients with chronic heart failure. European Journal of Cardiovascular Nursing, 12, 69-77.

Barra, E. (2014). Apoyo social, estrés y salud. Psicología y Salud, 14, 237-243.

Beach, S. R., Katz, J., Kim, S., y Brody, G. H. (2003). Prospective effects of marital satisfaction on depressive symptoms in established marriages: A dyadic model. Journal of Social and Personal Relationships, 20,355-371.

Bentler, P. M., y Weeks, D. G. (1980). Linear structural equations with latent variables. Psychometrika, 45, 289-308.

Bertera, E. M. (2005). Mental health in US adults: The role of positive social support and social negativity in personal relationships. Journal of Social and Personal Relationships, 22(1), 33-48.

Brislin, R. W. (1970) Back-translation for cross-cultural research. Journal of Cross-Cultural Psychology, 1, 185-216.

Brooks, K. P., y Dunkel-Schetter, C. (2011). Social negativity and health: Conceptual and measurement issues. Social and Personality Psychology Compass, 5, 904-918.

Carpenter, L. L., Gawuga, C. E., Tyrka, A. R., Lee, J. K., Anderson, G. M., y Price, L. H. (2010). Association between plasma IL-6 response to acute stress and early-life adversity in healthy adults. Neuropsychopharmacology, 35, 2617-2623.

De Vogli, R., Chandola, T., y Marmot, M. G. (2007). Negative aspects of close relationships and heart disease. Archives of Internal Medicine, 167, 1951-1957.

Finch, J. F., Okun, M. A., Pool, G. J., \&Ruehlman, L. S. (1999). A comparison of the influence of conflictual and supportive social interactions on psychological distress. Journal of Personality, 67(4), 581-621.

Ibarra-Rovillard, M. S., y Kuiper, N. A. (2011). Social support and social negativity findings in depression: Perceived responsiveness to basic psychological needs. Clinical Psychology Review, 31, 342-352.

Miller, G. E., Chen, E., \& Zhou, E. S. (2007). If it goes up, must it come down? Chronic stress and the hypothalamic-pituitary-adrenocortical axis in humans. Psychological Bulletin, 133(1), 25-45.

Newsom, J. T., Mahan, T. L., Rook, K. S., y Krause, N. (2008). Stable negative social exchanges and health. Health Psychology, 27, 78.

Newsom, J. T., Nishishiba, M., Morgan, D. L., \& Rook, K. S. (2003). The relative importance of three domains of positive and negative social exchanges: a longitudinal model with comparable measures. Psychology and Aging, 18(4), 746.

Newsom, J. T., Rook, K. S., Nishishiba, M., Sorkin, D. H., y Mahan, T. L. (2005). Understanding the relative importance of positive and negative social exchanges: Examining specific domains and appraisals.The Journals of Gerontology Series B: Psychological Sciences and Social Sciences, 60, P304-P312.
Ortiz, M. S., Baeza-Rivera, M. J., y Myers, H. F. (2013). Propiedades psicométricas de la escala de estrés para diabéticos en una muestra de pacientes diabéticos tipo II chilenos. Terapia Psicológica, 31, 281-286.

Ortiz, M. S., Gómez-Pérez, D., Cancino, M., Barrera-Herrera, A. (2016). Validación de la version en Español de la Escala de Optimismo Disposicional (LOT-R) en una muestra Chilena de estudiantes universitarios. Terapia Psicológica, 34(1), 53-58.

Ortiz, M. S., Willey, J. F., \& Chiang, J. J. (2014). How stress gets under the skin o cómo el estrés psicológico se introduce bajo la piel. Revista Médica de Chile, 142(6), 767-774.

Rook, K. (2014). The health effects of negative social exchanges in later life. Generations, 38, 15-23.

Ruehlman, L. S., y Karoly, P. (1991). With a little flak from my friends: Development and preliminary validation of the Test of Negative Social Exchange (TENSE). Psychological Assessment: A Journal of Consulting and Clinical Psychology, 3, 97-104

Schreiber, J. B., Nora, A., Stage, F. K., Barlow, E. A., \& King, J. (2006). Reporting structural equation modeling and confirmatory factor analysis results: A review. The Journal of Educational Research, 99(6), 323-338.

Smith, T. W., Uchino, B. N., Berg, C. A., Florsheim, P., Pearce, G., Hawkins, M., ... y Olsen-Cerny, C. (2009). Conflict and collaboration in middle-aged and older couples: II. Cardiovascular reactivity during marital interaction. Psychology and Aging, 24, 274.

Taylor, S. E., Lehman, B. J., Kiefe, C. I., y Seeman, T. E. (2006). Relationship of early life stress and psychological functioning to adult C-reactive protein in the coronary artery risk development in young adults study. Biological Psychiatry, 60, 819-824.

Vinokur, A. D., y Van Ryn, M. (1993). Social support and undermining in close relationships: their independent effects on the mental health of unemployed persons. Journal of Personality and Social Psychology, 65,350 .

Walen, H. R., y Lachman, M. E. (2000). Social support and strain from partner, family, and friends: Costs and benefits for men and women in adulthood. Journal of Social and Personal Relationships, 17, 5-30. 


\section{Anexo 1. Escala de Negatividad Social de Newsom et al. 2005}

\section{Instrucciones.}

Lee con atención cada una de las siguientes afirmaciones e indica qué tan frecuentemente te han ocurrido en el último mes. Para tus respuestas usa las siguientes opciones.

$1=$ nunca; 2 = casi nunca; 3 = algunas veces; $4=$ casi siempre; $5=$ siempre

Durante el mes pasado ¿cuán frecuentemente la gente que conoces?

- Te dio consejos que no necesitabas (no pediste)

- Cuestionó tus decisiones

- Interfirió en tus asuntos personales sin que lo pidieras

- Te defraudó justo cuando necesitabas ayuda

- Te molestó pidiéndote mucha ayuda

- Falló en darte ayuda con la cual contabas

- Te excluyó de una actividad en la que te hubiese gustado participar

- Te ignoró

- Te dedicó poco tiempo

- Fue poco considerado contigo

- Se enojó contigo

- Fue crítico respecto de alguna de tus preocupaciones 